\title{
Application of relay puncture technique in treating patients with complicated lower extremity arterial diseases
}

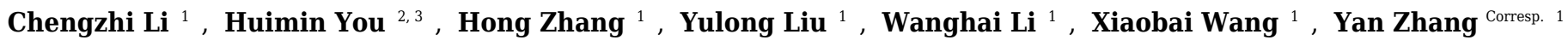 \\ 1 Department of Interventional Radiology and Vascular Surgery, The First Affiliated Hospital of Jinan University, Guangzhou, Guangdong, China \\ 2 Department of endocrinology, The Fifth Affiliated Hospital of Guangzhou Medical University, Guangzhou, Guangdong, China \\ 3 Medical Imaging Center, The First Affiliated Hospital of Jinan University, Guangzhou, Guangdong, China \\ Corresponding Author: Yan Zhang \\ Email address: dsazy@163.com
}

Objective: This study aimed to introduce and evaluate the safety and efficacy of the relay puncture technique in patients with complicated lower extremity arterial diseases.

Methods: A total of 21 patients (16 male and five female patients; median age: 70 years old), who had suffered from lower extremity arterial diseases between December 2014 and July 2017, were retrospectively collected. For all patients, the contralateral femoral artery was not available for puncture access, and the length of the devices was too short for the brachial artery approach. Therefore, the relay puncture technique, in which the first puncture was performed on the brachial artery, followed by an anterograde puncture on the femoral artery, was used to accomplish the endovascular therapy. Percutaneous transluminal angioplasty ( PTA) and/or percutaneous transluminal stenting ( PTS) was usedto assess the efficacy of the relay puncture technique. The ankle - brachial index (ABI) and Rutherford Clinical Classification (RCC) were used to evaluate the improvement of symptoms after treatment. Patients were followed up for 1, 3, 6 and 12 months, and annually (mean: 16.6 months) after discharge.

Results: The relay puncture treatment had a 100\% technical success rate, and immediately decreased the ischemic symptoms of patients after the procedure. The ABI significantly increased from $0.33 \pm 0.18$ to $0.75 \pm 0.21$ at the 1 -year follow-up time point $(P<0.05)$. No serious complications occurred during the follow-up period.

The 1-year primary patency rate was $71.43 \%$.

Conclusion : The relay puncture technique is a feasible technique in the hands of experienced and skilled equipment operators for the treatment of lower extremity arterial diseases, when the contralateral femoral artery is not available for puncture, and the length of the device is too short to treat the distal lesion of the femoral artery and popliteal artery through the brachial artery approach. 


\section{Application of relay puncture technique in treating patients with complicated}

\section{2 lower extremity arterial diseases}

4 Authors' information

5 1. Chengzhi Li, Department of Interventional Radiology and Vascular Surgery, The First

6 Affiliated Hospital of Jinan University, Guangzhou, China, 510630. E-mail:

7 showshow009@163.com. Tel: 008615918763184.

8 2. Huimin You, First unit: Department of endocrinology, The Fifth Affiliated Hospital of

9 Guangzhou medical university, Guangzhou, China, 510700. Second unit: Medical imaging 10 center, The First Affiliated Hospital of Jinan University, Guangzhou, China, 510630. E-mail:

11 youhuimin110@163.com. Tel: 008613423602633.

12 3. Hong Zhang, Department of Interventional Radiology and Vascular Surgery, The First

13 Affiliated Hospital of Jinan University, Guangzhou, China, 510630. E-mail:

14 zhangboda@sohu.com. Tel: 008613711526778.

15 4. Yulong Liu, Department of Interventional Radiology and Vascular Surgery, The First 16 Affiliated Hospital of Jinan University, Guangzhou, China, 510630. E-mail: 22646911@qq.com.

17 Tel: 008615099950532.

18 5. Wanghai Li, Department of Interventional Radiology and Vascular Surgery, The First

19 Affiliated Hospital of Jinan University, Guangzhou, China, 510630. E-mail:

20 1291119705@qq.com. Tel:008613247398113.

21 6. Xiaobai Wang, Department of Interventional Radiology and Vascular Surgery, The First

22 Affiliated Hospital of Jinan University, Guangzhou, China, 510630. E-mail:

23 xiaobaiwang@163.com. Tel: 008613392692251. 
24 7. Yan Zhang, Department of Interventional Radiology and Vascular Surgery, The First

25 Affiliated Hospital of Jinan University, Guangzhou, China, 510630. E-mail: dsazy@163.com.

26 Tel: 008613922206007. (Corresponding author)

27

28 The first two authors contributed equally to this work.

29

30 Original article

31 Word count: 4367

32 Declarations of interest: None

33 Short title: Application of relay puncture technique 


\section{Abstract}

Objective: This study aimed to introduce and evaluate the safety and efficacy of the relay puncture technique in patients with complicated lower extremity arterial diseases. who suffered from lower extremity arterial diseases between December 2014 and July 2017, were retrospectively collected. For all patients, the contralateral femoral artery was not available for puncture access, and the length of the devices was too short for the brachial artery approach. Therefore, the relay puncture technique, in which the first puncture was performed on the brachial artery, followed by an anterograde puncture on the femoral artery, was used to accomplish the endovascular therapy. Percutaneous transluminal angioplasty (PTA) and/or percutaneous transluminal stenting (PTS) was used to assess the efficacy of the relay puncture technique. The ankle-brachial index (ABI) and Rutherford Clinical Classification (RCC) were used to evaluate the improvement of symptoms after treatment. Patients were followed up for 1 , 3, 6 and 12 months, and annually (mean: 16.6 months) after discharge.

Results: The relay puncture treatment had a $100 \%$ technical success rate, and immediately decreased the ischemic symptoms of patients after the procedure. The ABI significantly increased from $0.33 \pm 0.18$ to $0.75 \pm 0.21$ at the 1 -year follow-up time point $(P<0.05)$. No serious complications occurred during the follow-up period. The 1-year primary patency rate was 
$71.43 \%$.

Conclusion: The relay puncture technique is a feasible technique in the hands of experienced and skilled equipment operators for the treatment of lower extremity arterial diseases, when the contralateral femoral artery is not available for puncture, and the length of the device is too short to treat the distal lesion of the femoral artery and popliteal artery through the brachial artery approach.

Keywords: Antegrade femoral artery puncture, lower extremity arterial disease, endovascular treatment

\section{Introduction}

Advances in endovascular technologies in recent years have offered more options for patients with lower extremity arterial diseases and expanded the indications for endovascular therapy [1]. However, in some complex situations when the contralateral femoral artery cannot be punctured, such as when cover stents are implanted on the entire contralateral femoral artery, or the length of the device is too short to treat the distal end of the femoral and popliteal artery through the brachial artery approach, a simple endovascular technique cannot solve the problem [2]. For these situations, a hybrid procedure, such as endovascular and open surgery, is usually an appropriate choice [3]. However, some disadvantages of the hybrid procedure include more complicated surgery requiring skilled clinicians to achieve appreciable outcomes, and the requirement of an operating room for performing this procedure [4]. Therefore, it is imperative to determine new techniques that can replace hybrid surgery with acceptable efficacy, safety and simplicity in the treatment of complicated lower extremity diseases.

In the present study, a simple novel endovascular technique, which was named, the relay 
93 puncture technique, was employed, in which the first puncture was performed on the brachial 94 artery, followed by an anterograde puncture of the femoral artery. The investigators were able to

95 treat the femoral artery lesions through the brachial artery, while the investigators were able to 96 treat lesions distal to the popliteal artery through the femoral artery. The relay puncture 97 technique was used to treat patients whose contralateral access was not available, since these 98 patients had ipsilateral or contralateral iliac and common femoral axis stenosis or occlusions, a very angulated aortic bifurcation of the iliac, a puncture site with heavy calcifications or a stent, infection of the groin, and a cross-over maneuver in the setting of the bifurcated aortic graft or

101

102

103

104

105

106

107

108

109

110

111

112

113

114

115

pre-existing iliac kissing stents. The safety and efficacy of the relay puncture technique was evaluated in the present study.

\section{Materials and Methods}

\subsection{Eligibility}

A total of 21 patients (16 male and five female patients), who were within 48-81 years old (median age: 68.5 years old) and suffered from lower extremity arterial disease, were collected in our hospital between December 2014 and July 2017. The diagnosis of each patient was confirmed by ultrasonography and computed tomography angiography (CTA) before the treatment. All patients had clinical symptoms of critical chronic lower extremity ischemia. The ankle brachial index (ABI) and Rutherford Clinical Classification (RCC) before and after the operation were used to evaluate the improvement of the lower extremity ischemia of each patient after the procedure. According to the TASC classification, all patients in the present study were type D, and all patients declined to have open surgery, which was probably due to fear of major trauma, advanced age, and/or their family's wishes. 


\subsection{Institutional review}

The experimental protocol of the present study complied with the principles outlined in the Declaration of Helsinki, and was approved by the Ethics Committee of our institution. All subjects provided a signed informed consent.

\subsection{Procedures of the relay puncture technique}

The patient was placed in the supine position and hypodermically administered with heparin (5,000 units) after a 5-French sheath (Terumo, Japan or Cook, USA) was inserted through the left brachial artery, followed by repeated heparin administration in prolonged procedures. After endovascular treatment of the iliac, femoral, or popliteal artery, the $5 \mathrm{~F}$ sheath was changed to a 6F sheath, which is a 90-cm-long armored introducer sheath (COOK, USA), in order to achieve adequate support and strengthen the control of the catheter. Then, a 0.035-inch J-type guidewire (Terumo, Japan) or 0.018-inch guidewire (V18; Boston Scientific, USA) supported by a 5F vertebral artery angiography catheter (Cordis, Johnson \& Johnson, USA) was navigated in the proximal common femoral artery.

In the present study, the relay puncture technique was used on the ipsilateral femoral artery in the following situations: the contralateral femoral artery could not be punctured (the stents were implanted in the entire contralateral femoral artery in seven patients); the stents were implanted into the aortic artery in the iliac lesion (four patients); there was abdominal aortic aneurysm after the endovascular repair (five patients), which made it difficult for the devices to cross over the bifurcation; the amputation of the contralateral leg did not allow the on-site puncture of the femoral artery (five patients). These five patients only required the recanalization of the contralateral lower extremity artery due to cost problems, and the length of the devices was too short to treat the distal end of the femoral artery and popliteal artery through the brachial 
139 artery approach. The lesions in all these patients involved the area of the upper inguinal, through

140 which an antegrade femoral puncture could not be successfully performed.

141 The detailed procedures of the puncture technique are described, as follows. After the 142 recanalization of the proximal part of the femoral artery, the wire was left in place as a marker.

143 Then, an antegrade puncture was performed at the groin under fluoroscopic guidance, and a 5F

144 10-cm sheath was inserted. Then, another J-type 0.035-inch guide wire or V18 was used to cross 145 the femoropopliteal lesion, including the tibial disease, followed by a 6-8 $\mathrm{mm}$ diameter classical 146 percutaneous transluminal angioplasty (PTA) balloon (Inpact, Medtronic, Minneapolis, MN, 147 USA) in the iliofemoral segment, a 5-6 $\mathrm{mm}$ diameter balloon in the femoropopliteal, and a 2-3 148 149 150 $\mathrm{mm}$ diameter balloon in arteries below the knee, and stenting. The balloon inflation times varied within 60-300 seconds at nominal pressure. Subintimal angioplasty was also used, and subintimal arterial flossing with the antegrade-retrograde intervention (SAFARI) technique was used for re-entry. Immediately after the vascularization procedure, the sheath was removed from the femoral artery, and a 6-French vascular closer (Exoseal, Cordis; Johnson \& Johnson, USA) was used to stop the bleeding of the punctured site. The median compression time was five minutes (range: 3-10 minutes). The angioplasty inside the punctured site with a 6-mm balloon was helpful in stopping the bleeding after the sheath was removed (6/16). Finally, PTA and percutaneous transluminal stenting (PTS) were performed on the proximal part of the femoral artery and iliac artery through the left brachial artery approach without any open surgery. After the completion of all these procedures, the sheath in the left brachial artery was withdrawn. All the above-mentioned endovascular procedures were performed by experienced interventional radiologists in our center.

\subsection{Endpoints and follow up}


162

163

164

165

166

167

168

169

170

171

172

173

174

175

176

177

178

179

180

181

182

183

184

The primary endpoint was the efficacy of the relay puncture technique, as indicated by the acute technical success and post-procedure clinical improvement of critical limb ischemia (CLI) symptoms. The secondary endpoint was the safety of this technique, as indicated by the number or severity of complications associated with this procedure.

Color Doppler ultrasonography and/or CT angiography were performed at 1, 3, 6 and 12 months, and annually, or when symptoms recurred after discharge. Antiplatelet therapy was administered during the whole life of each patient after treatment to prevent restenosis.

\subsection{Statistical analysis}

Data were presented as mean \pm standard deviation. All statistical analyses were performed using SPSS software version 22.0 for PC (SPSS Inc., Chicago, IL, USA). Least significant difference $t$-test was used to compare the ABI before and after treatment. A $P$-value $<0.05$ was considered statistically significant.

\section{Results}

\subsection{Short term outcomes}

A total of 21 patients (16 male and five female patients; median age: 70 years old), who suffered from lower extremity arterial diseases from December 2014 to July 2017, were retrospectively collected. All patients were diagnosed with lower extremity arterial disease before treatment. Preoperative CTA clearly revealed the occlusive lesions of the lower extremity arteries (Figs. 1 and 2). For all these patients, the contralateral femoral artery was not available for puncture access, and the length of the devices was too short for the brachial artery approach. Among these patients, seven patients had cover stents on the entire contralateral femoral artery, four patients had kissing stents in the aortoiliac lesion, five patients experienced endovascular 
185 repair of abdominal aortic aneurysm, and five patients underwent amputation of the contralateral

186 leg due to ischemia. After a detailed preoperative discussion and project design, all patients

187 underwent the relay puncture procedure, and technical success was achieved in all occlusive

188 arteries (Figs. 3 and 4). Furthermore, no complication occurred during the treatment. A total of 18987 stents (median: 3) were implanted in 21 patients. Perioperative complications included 190 hematoma (one patient) at the brachial artery puncture site and heart failure (one patient) before 191 discharge. These two patients were successfully treated and discharged after treatment. Moreover, 192 the postoperative ABI significantly increased $(0.33 \pm 0.18$ vs. $0.75 \pm 0.21, P<0.05)$. 193 Correspondingly, the ischemia symptoms were immediately alleviated after the treatment, as 194 evaluated by RCC (Table 1).

195 3.2. Long-term outcomes

196 All patients were followed up for 4-28 months (mean: 16.6 months). During the follow-up 197 period, no serious complications were observed. Color Doppler ultrasonography revealed that the 198 1-year primary patency rate was $71.43 \%(15 / 21)$. The changes in ABI of these patients during 199 the follow up period are summarized in Table 1. suffered from lower extremity arterial disease have been successfully treated with endovascular repair [5]. The SAFARI technique has also broadened the indications for endovascular therapy in patients with lower extremity arterial disease. However, in some complex situations, lesions could not be merely cured by endovascular treatment, even with the SAFARI technique. The 207 patients presented in the present study had a complicated pathophysiology in diseased lower 
208

209

210

211

212

213

214

215

216

217

218

219

220

221

222

223

224

225

226

227

228

229

230

extremities, and this could not be treated by merely traditional endovascular methods. Therefore, the two position's puncture method was applied to complete the endovascular treatment for these patients. The investigators named this method the relay puncture technique. The present study suggests that the relay puncture technique has appreciable safety and efficacy.

\subsection{Relay puncture technique}

Patients in the present study did not have access on the contralateral femoral artery due to different reasons. The hybrid operation, which is a combined endovascular and surgical technique, was used as an alternative to laparotomy to correct inflow lesions in patients with multilevel arterial diseases [6], and this has achieved appreciable patency rates and technical success rates [7]. However, the hybrid surgery exhibited several disadvantages, including the requirement of highly skilled surgeons (there are limited numbers of vascular surgeons), the need for an operation room (the cost of a hybrid operation room is high and cannot be sufficiently provided), and the potentially increased risk of complications and longer recovery period (including the risk correlated to further anesthesia) [8]. For example, one recent meta-analysis that compared the outcomes of the endovascular and open bypass treatment for TASC C-D aortoiliac occlusive disease reported more complications and greater 30-day mortality with open bypass [9].

In the present study, the relay puncture technique, which is a new puncture method with different approaches, was proposed to handle these complex vascular lesions. The word "relay" was used in this technique, because the antegrade puncture on the femoral artery after the brachial artery puncture was just like a relay race. This technique has been applied in 21 patients, and the short- and long- term outcomes appeared to be satisfactory, as evidenced by the findings that the technical success rate was $100 \%$, and that the primary long-term patency was $71.43 \%$, 
231 which were similar to those previously reported in studies on patients after endovascular 232 treatment [10].

For patients in the present study, there was no puncture site available on the lower extremity arteries. Thus, the investigators opted to puncture the left brachial artery first. However, all endovascular devices failed to reach the lesions at the distal part of the femoral and popliteal artery. The SAFARI technique should be able to establish a guidewire line through the whole lesion. However, the size of both the stent delivery catheter and balloon catheter were too big to be pulled into the arteries below the knee. This was the same with the atherectomy catheter and drug-coated balloon. Therefore, the SAFARI technique could not solve the problem of these patients in the present study, who had long lesions that involved the popliteal artery, or even those below the knee. Directly puncturing the stents on the contralateral femoral artery should have been a solution. However, that technology is not mature yet, and few studies have reported good long-term outcomes [12]. Hence, directly puncturing the contralateral femoral artery appeared as the only option [13]. Another approach that might solve the above-mentioned issue for these patients was to insert a large sheath with a drug coated balloon or debulking devices through the stent. This approach may damage the stent structure, subsequently accelerating the occurrence of stent rupture and/or restenosis within the stent. In addition, this technique cannot be used for patients with cover stents. Furthermore, in some cases, stents were implanted into the aortic artery in some aortic-iliac lesions, and it was difficult for the devices to cross over the iliac branches. Most importantly, all patients in the present study had lesions that involved the area of the upper inguinal, which could not be directly antegrade punctured through the femoral artery. Based on the above-mentioned analysis, the investigators decided to use the new relay puncture technique. 


\subsubsection{Puncture of the brachial artery.}

The antegrade approach using the trans-brachial access with long sheaths and dedicated catheters appeared to be the choice for aortoiliac occlusions with or without extension to the femoral-popliteal arterials. This approach offers better push ability, allows access to both iliac arteries, and enables the reentry at the femoral artery to be managed more easily and safely, when compared with the retrograde approach [14]. Although this involved some risks and technical challenges, the investigators continued to choose this approach for patients in the present study, because they had no other puncture channels. It turned out that this antegrade approach was successfully performed in the present study.

\subsubsection{Antegrade puncture of the superficial femoral artery}

At present, performing an antegrade puncture has become a routine in endovascular treatment for patients suffering from infra-inguinal lower extremity arterial disease [15]. Recent studies have shown that antegrade access via the superficial femoral artery (SFA) has practical advantage over classical access via the common femoral artery (CFA), in terms of shorter access time, quicker fluoroscopy time, and higher success rate $[16 ; 17]$. In the present study, before the SFA was punctured, the guidewire recanalized the vessel and reached the target. Therefore, under fluoroscopy, the antegrade procedure to puncture the guidewire was safe with fewer complications. However, the number of attempts of reentry procedures with the guidewire should be limited to avoid extending the dissection. Regardless of whether it was successful or not, the guidewire guided the antegrade puncture performed to the SFA. In addition, this approach should be performed at the same axis, while the former guidewire should be performed in the lumen, in order to allow these two guide wires to link together. Basically, the first wire created an access, while the second wire took over the treatment of femoral-popliteal lesions, as well as arterial 
277 lesions below the knee. The relay approach not only recanalized these infra-inguinal diseases, 278 but also overcame the short-shaft, further increasing the pushing ability and maneuverability of 279 the wire, and the probability of reentry, especially near the popliteal artery. Moreover, the latter 280 wire may be used to reconstruct the arterials below the knee.

281

282

283

284

285

286

287

288

289

290

291

292

293

294

295

296

297

298

299

\subsection{Application of puncture closure devices}

Endovascular treatment has been shown to be associated with several complications. For instance, the incidence of hematoma can reach up to $6 \%$ for patients undergoing brachial puncture [18], and the incidence of complications following the antegrade puncture of SFA can reach up to $9 \%$ [19]. However, the use of closure devices exhibited a significant tendency to decrease these complications [20]. Consistent with these reports, in the present study, the median compression time was only five minutes (3-10 minutes), even in the presence of heparin, which was shorter than the traditional compression time for hemostasis. Furthermore, using the Exoseal device to close the antegrade puncture hole on the femoral artery, endovascular therapy could be quickly continued without bleeding in the present study. A variety of arterial closure devices have been introduced with the aim of reducing bleeding risk. However, all were indicated for femoral use only [21]. In the present study, Exoseal was used for brachial artery puncture site closure, which is a safe technique with an acceptable rate of complications (6\%). Thus, no clinical intervention was required.

\section{Limitations of the present study}

The present study was a retrospective study with a limited sample size. Hence, the present findings need to be further corroborated in large cohort studies in the future. In addition, the procedures for the relay puncture technique remains complex, and needs to be performed by an 
300 experienced doctor.

301

302

303

\section{Conclusion}

304

The present study described the modalities of the "relay puncture" technique. The 305 preliminary results of the present study revealed that this may be a feasible technique in the hands of experienced and skilled operators for the treatment of patients with lower extremity arterial diseases, when the contralateral femoral artery cannot be punctured and the length of the devices is too short to treat the distal lesion of the femoral and popliteal artery through the brachial artery approach.

\section{Acknowledgements}

312 None.

313

\section{Conflict of interests}

315 The authors declare that there is no conflict of interest. 
[1] Goodney PP1, Beck AW, Nagle J, Welch HG, Zwolak RM. 2009. National trends in lower extremity bypass surgery, endovascular interventions, and major amputations. J Vasc Surg, 320 50(1):54-60.

[2] Yilmaz S, Sindel T, Yegin A, Lüleci E. 2003. Subintimal angioplasty of long superficial femoral artery occlusions. J Vasc Interv Radiol, 14(8): 997-1010.

[3] Antoniou GA1, Sfyroeras GS, Karathanos C, et al. 2009. Hybrid endovascular and open treatment of severe multilevel lower extremity arterial disease. Eur J Vasc Endovasc Surg, 325 38(5):616-22.

[4] Murakami A. 2018. Hybrid Operations in Patients with Peripheral Arterial Disease. Ann 327 Vasc Dis, 11(1): 57-65.

[5] Hendricks NJ, Sabri SS. 2014. Subintimal arterial flossing with antegrade-retrograde intervention (SAFARI) for subintimal recanalization to treat chronic critical limb ischemia. Techniques in Vascular and Interventional Radiology, 17(3): 203-210.

[6] Madera FA, Orecchia PM, Razzino RA, Razzino J, Calcagno D. 1997. Balloon angioplasty 
by vascular surgeons. Am J Surg, 174(2): 152-6.

333 [7] Mousa A, Abdel-Hamid M, Ewida A, Saad M, Sahrabi A. 2010. Combined percutaneous

334 endovascular iliac angioplasty and infrainguinal surgical revascularization for chronic lower 335 extremity ischemia: preliminary result. Vascular, 18(2): 71-6.

[8] Tsuyoshi Kaneko, Michael J. Davidson. 2014. Use of the hybrid operating room in cardiovascular medicine. Circulation, 130:910-917.

[9] Thomas T. Tsai, Thomas F. Rehring, R. Kevin Rogers, Susan M. Shetterly, Nicole M.

Wagner, Rajan Gupta, et al. 2015. The contemporary safety and effectiveness of lower extremity bypass surgery and peripheral endovascular interventions in the treatment of symptomatic peripheral arterial disease. Circulation, 132:1999-2011.

[10] Klein AJ, Ross CB. 2016. Endovascular treatment of lower extremity peripheral arterial 343 disease. Trends Cardiovasc Med, 26(6): 495-512.

[11] Bulut T, Oosterhof-Berktas R, Geelkerken RH, Brusse-Keizer M, Stassen EJ, Kolkman JJ. 2017. Long-Term Results of Endovascular Treatment of Atherosclerotic Stenoses or Occlusions

346 of the Coeliac and Superior Mesenteric Artery in Patients with Mesenteric Ischaemia. Eur J Vasc 347

Endovasc Surg. 53(4):583-590. 
348 [12] Jongkind V, Akkersdijk GJ, Yeung KK, Wisselink W. 2010. A systematic review of

349 endovascular treatment of extensive aortoiliac occlusive disease. J Vasc Surg, 52(5): 1376-83.

350 [13] Palena LM, Manzi M. 2013. Direct stent puncture technique for intraluminal stent

351 recanalization in the superficial femoral and popliteal arteries in-stent occlusion: outcomes from

352 a prospective clinical analysis of diabetics with critical limb ischemia. Cardiovasc Revasc Med, 353 14(4): 203-6.

354 [14] Millon A, Della SN, Brizzi V, Arsicot M, Boudjelit T, Herail J, et al. 2015. The Antegrade 355 Approach Using Transbrachial Access Improves Technical Success Rate of Endovascular 356 Recanalization of TASC C-D Aortoiliac Occlusion in Case of Failed Femoral Access. Ann Vasc 357 Surg, 29(7): 1346-52.

358 [15] Nice C, Timmons G, Bartholemew P, Uberoi R. 2003. Retrograde vs. antegrade puncture 359 for infra-inguinal angioplasty. Cardiovasc Intervent Radiol, 26(4): 370-4. femoral artery. Eur Radiol, 21(6): 1323-8. 
364 versus common femoral artery punctures for infrainguinal occlusive disease. J Vasc Interv 365 Radiol, 23(9): 1160-4.

366 [18] Alvarez-Tostado JA, Moise MA, Bena JF, Pavkov ML, Greenberg RK, Clair DG, et al. 367 2009. The brachial artery: a critical access for endovascular procedures. J Vasc Surg, 49(2): 37836885.

[19] Mlekusch W, Minar E, Dick P, Sabeti S, Bartok A, Haumer M, et al. 2008. Access site management after peripheral percutaneous transluminal procedures: Neptune pad compared with conventional manual compression. Radiology, 249(3): 1058-63.

[20] Andre zeit A, Schoch E, Reischauer C, Hergan K, Jenelten R, Binkert CA. 2014. Comparison of a $21 \mathrm{G}$ micropuncture needle and a regular $19 \mathrm{G}$ access needle for antegrade arterial access into the superficial femoral artery. Cardiovasc Intervent Radiol, 37(2): 343-7. closure devices: a comparative overview. Curr Probl Diagn Radiol. 38(1):33-43. 
Figure legends

Figure 1 The CTA image for patient 1 before the operation. This image shows that the right SFA was totally occluded, and cover stents were implanted in the entire left CIA to SFA, which was unobstructed. The left femoral artery could not be punctured due to the cover stents.

Figure 2 CTA image for patient 2 before the operation. The image shows that the left SFA was totally occluded. The right leg had a high position amputation one year ago, and the right iliac artery was also occluded. The right femoral artery could not be punctured due to the amputation, and the patient only required recanalization due to economic issues.

Figure 3 Images showing the technical steps of the relay puncture technique performed for

patient 1. (a-c) Images of the angiography before treatment show the occlusion of the right SFA and the cover stents of the left side. $(\mathrm{d}, \mathrm{e})$ The images show that the recanalization was performed to the proximal part of right SFA through the left brachial access, and the PTA was first performed. (f, g) The images show that the relay puncture technique (antegrade puncture) was performed to the right femoral artery under fluoroscopy and the guidance of the guidewire, which was just recanalized. $(h, i)$ The images show that the recanalization was further performed 400 to the distal part of the right SFA and the arteries below the knee after the relay puncture. $(\mathrm{j}-1)$ 
401 The images show the angiography after endovascular treatment and the removal of the sheath in 402 the right femoral artery. Note that the recanalization was successful without contrast medium 403 overflow.

404

405 Figure 4 The images show the relay puncture technique used for patient 2 . (a-d) The 406 angiography before treatment shows the occlusion of the left SFA and right iliac to the femoral 407 artery, and that the right femoral artery could not be punctured due to the amputation. (e-h) 408 Similar to Figure 3, the angiography shows that the left SFA was successfully recanalized after 409 the application of the relay puncture technique. 


\section{Figure 1}

The CTA image for patient 1 before the operation

This image shows that the right SFA was totally occluded, and cover stents were implanted in the entire left CIA to SFA, which was unobstructed. The left femoral artery could not be punctured due to cover stents.

*Note: Auto Gamma Correction was used for the image. This only affects the reviewing manuscript. See original source image if needed for review. 


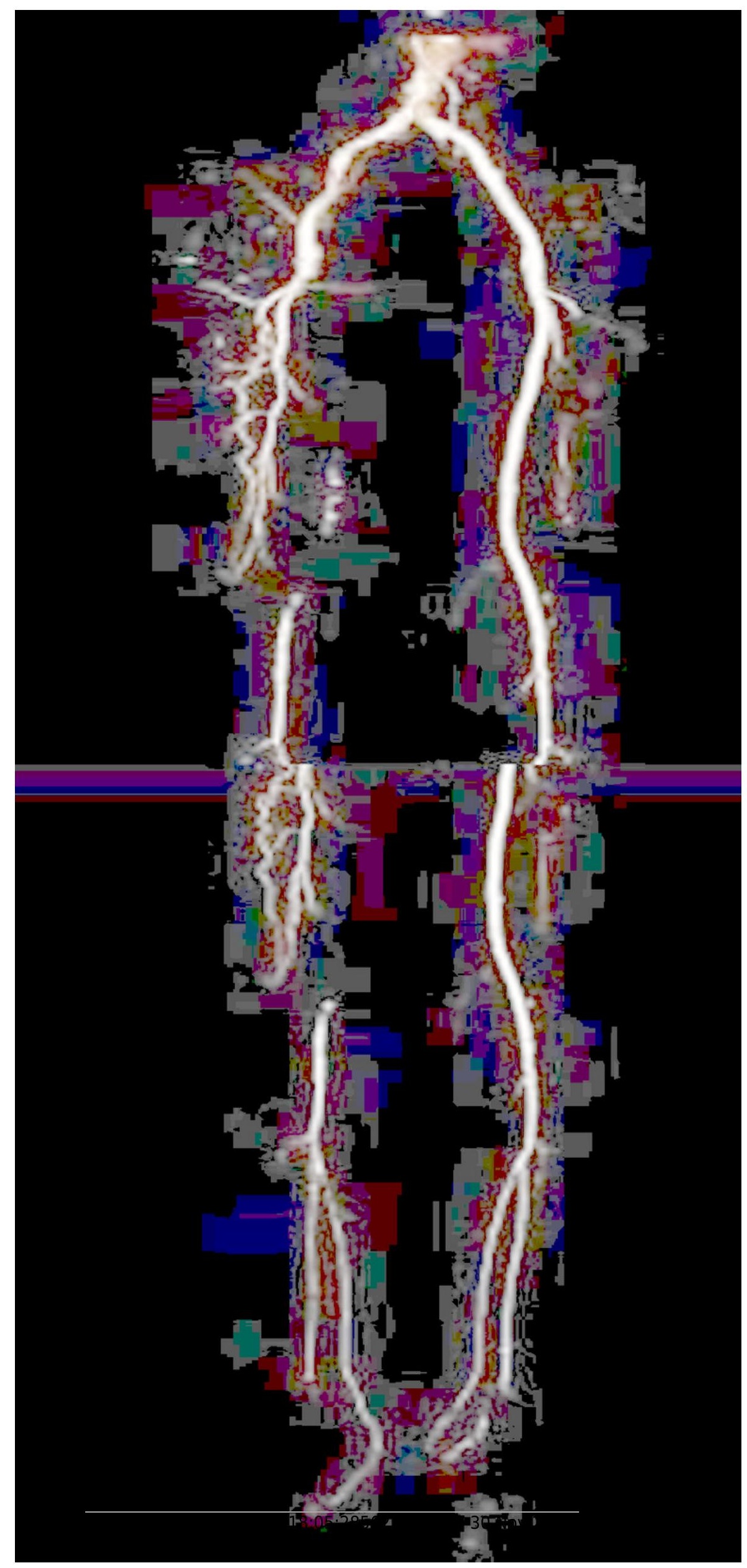




\section{Figure 2}

CTA image for patient 2 before the operation

The image shows that the left SFA was totally occluded. The right leg had a high position amputation one year ago, and the right iliac artery was also occluded. The right femoral artery could not be punctured due to the amputation, and the patient only required recanalization due to economic issues.

*Note: Auto Gamma Correction was used for the image. This only affects the reviewing manuscript. See original source image if needed for review. 


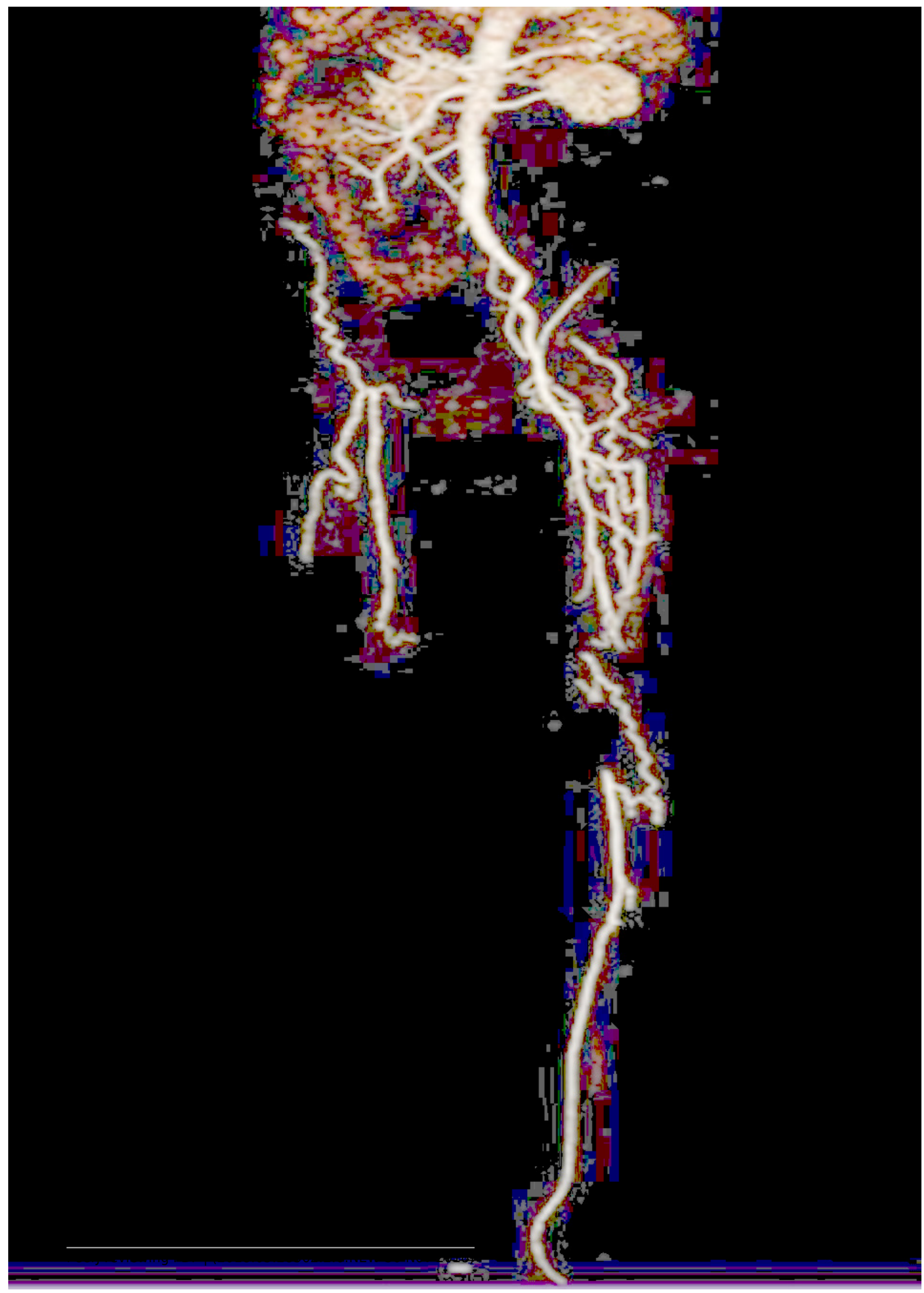




\section{Figure 3}

\section{Images showing the technical steps of the relaypuncture technique performed for patient 1}

(a-c) Images of the angiography before treatment show the occlusion of the right SFA and the cover stents of the left side. $(d, e)$ The images show that the recanalization was performed to the proximal part of right SFA through the left brachial access, and the PTA was first performed. $(f, g)$ The images show that the relay puncture technique (antegrade puncture) was performed to the right femoral artery under fluoroscopy and the guidance of the guide wire, which was just recanalized. $(h, i)$ The images show that the recanalization was further performed to the distal part of the right SFA and the arteries below the knee after the relay puncture. (j-I) The images show the angiography after endovascular treatment and the removal of the sheath in the right femoral artery. Note that the recanalization was successful without contrast medium overflow.

*Note: Auto Gamma Correction was used for the image. This only affects the reviewing manuscript. See original source image if needed for review.

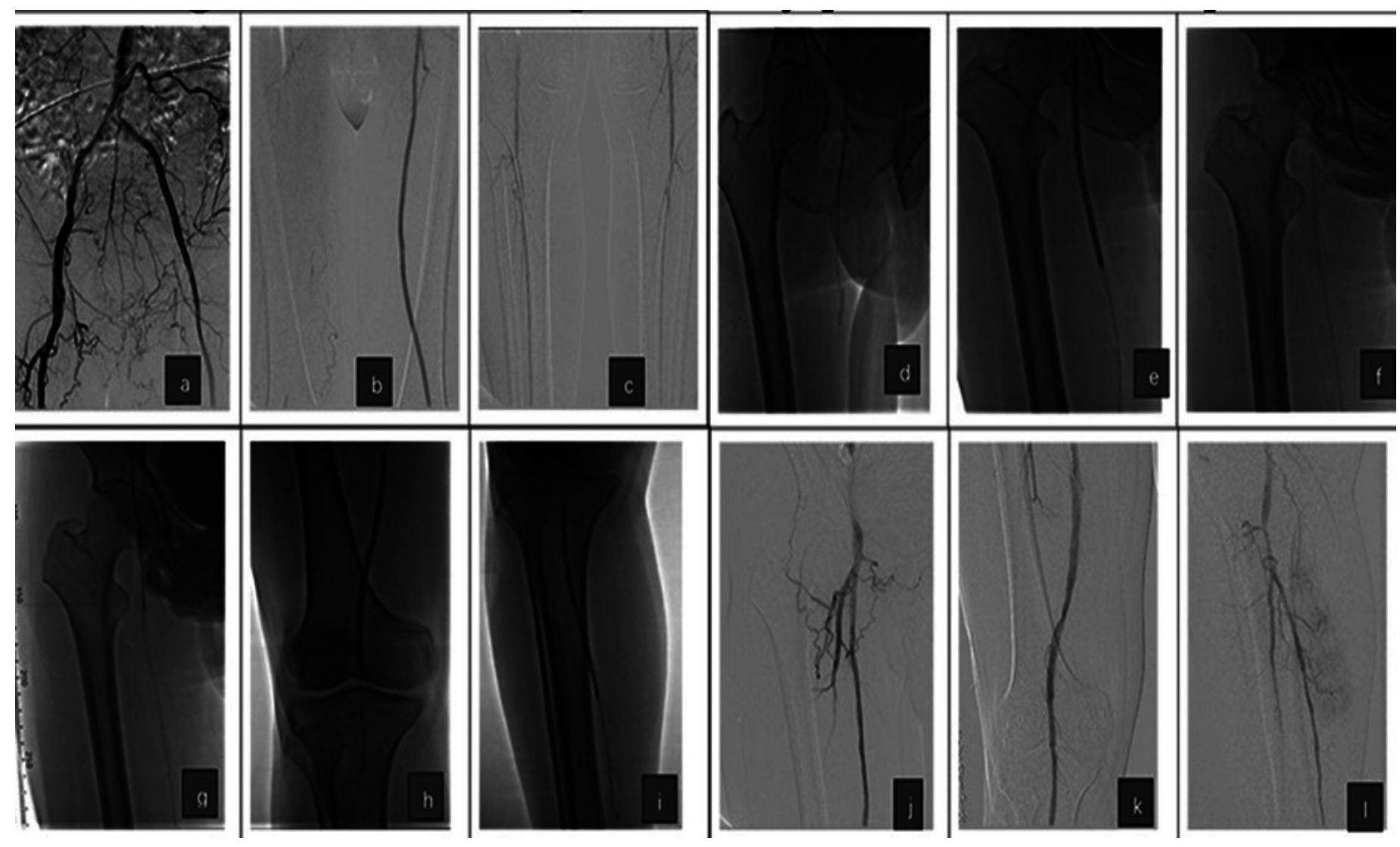




\section{Figure 4}

\section{The images show the relaypuncture technique used for patient 2}

(a-d) The angiography before treatment shows the occlusion of the left SFA and the right iliac to the femoral artery, and that the right femoral artery could not be punctured due to the amputation. (e-h) Similar to Figure 3, the angiography shows that the left SFA was successfully recanalized after the application of the relay puncture technique.

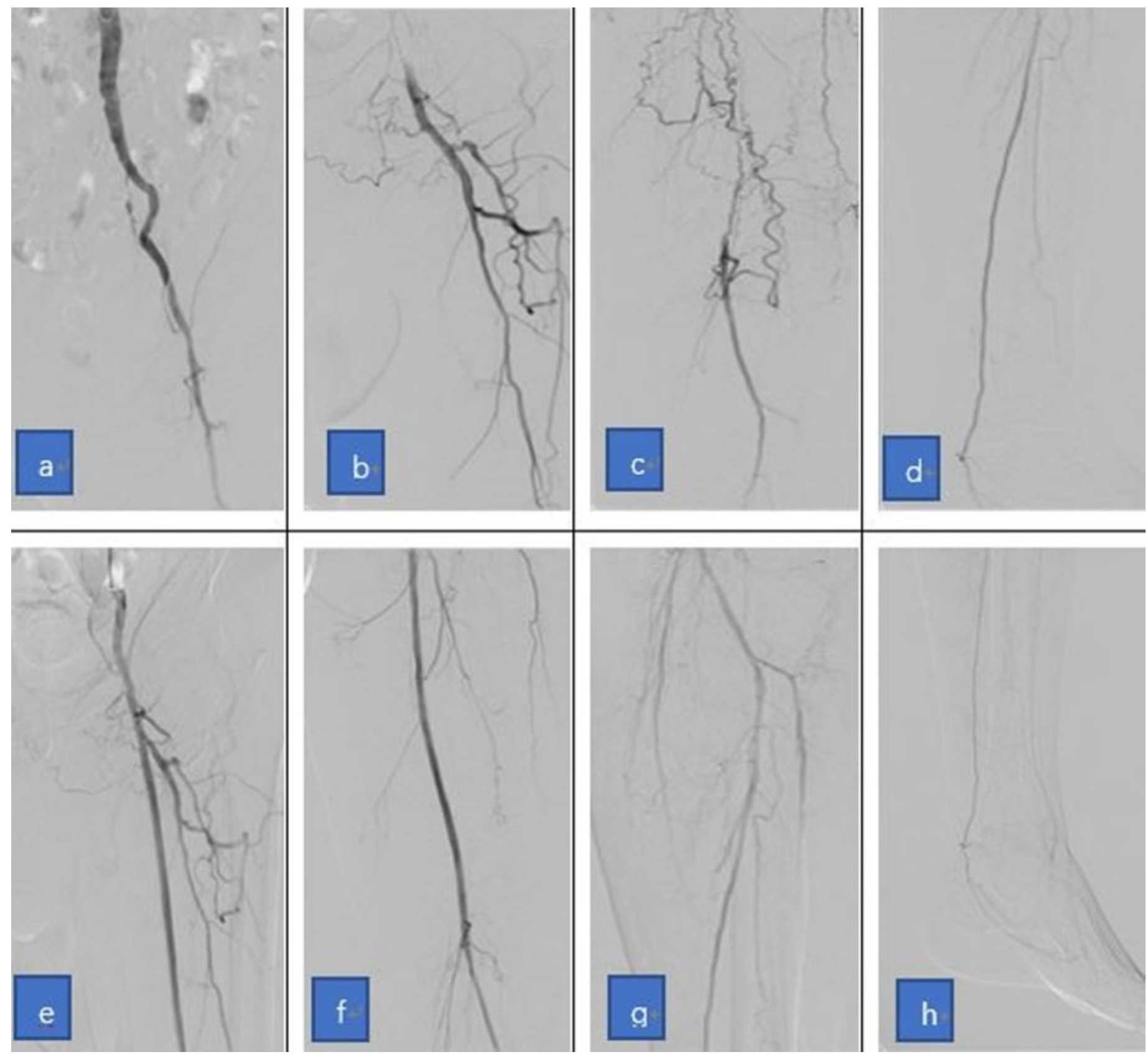




\section{Table 1 (on next page)}

Clinical symptom improvements of patients withinone year in the present study 
1 Table 1. Clinical symptom improvements of patients within one year in the present study

\begin{tabular}{|c|c|c|c|c|c|c|c|c|c|c|}
\hline \multirow[t]{2}{*}{$\begin{array}{l}\text { Patient } \\
\text { number }\end{array}$} & \multicolumn{2}{|c|}{$\begin{array}{l}\text { Before } \\
\text { treatment }\end{array}$} & \multicolumn{2}{|c|}{$\begin{array}{l}\text { Immediately } \\
\text { after treatment }\end{array}$} & \multicolumn{2}{|c|}{$\begin{array}{l}3 \text { months after } \\
\text { treatment }\end{array}$} & \multicolumn{2}{|c|}{$\begin{array}{l}6 \text { months after } \\
\text { treatment }\end{array}$} & \multicolumn{2}{|c|}{$\begin{array}{l}12 \text { months } \\
\text { after } \\
\text { treatment }\end{array}$} \\
\hline & RCC & ABI & RCC & ABI & RCC & ABI & RCC & ABI & RCC & ABI \\
\hline 1 & 6 & 0 & 0 & 0.55 & 0 & 0.63 & 1 & 0.58 & 4 & 0.42 \\
\hline 2 & 4 & 0.51 & 0 & 0.81 & 0 & 0.79 & 0 & 0.83 & 0 & 0.65 \\
\hline 3 & 4 & 0.48 & 0 & 0.93 & 0 & 0.90 & 0 & 0.88 & 0 & 0.92 \\
\hline 4 & 5 & 0.22 & 0 & 0.68 & 0 & 0.71 & 0 & 0.68 & 0 & 0.77 \\
\hline 5 & 4 & 0.36 & 0 & 0.99 & 0 & 1.03 & 0 & 0.98 & 0 & 0.95 \\
\hline 6 & 6 & 0 & 1 & 0.70 & 1 & 0.82 & 1 & 0.79 & 4 & 0.58 \\
\hline 7 & 3 & 0.60 & 0 & 1.08 & 0 & 1.05 & 0 & 1.02 & 0 & 0.98 \\
\hline 8 & 4 & 0.41 & 0 & 0.93 & 0 & 0.93 & 0 & 0.88 & 0 & 0.80 \\
\hline 9 & 5 & 0.45 & 0 & 1.12 & 1 & 1.05 & 0 & 1.02 & 0 & 0.95 \\
\hline 10 & 5 & 0.36 & 0 & 0.81 & 0 & 0.88 & 0 & 0.90 & 0 & 0.89 \\
\hline 11 & 4 & 0.72 & 0 & 1.10 & 0 & 1.00 & 0 & 0.98 & 0 & 0.96 \\
\hline 12 & 6 & 0 & 1 & 0.69 & 1 & 0.73 & 1 & 0.63 & 5 & 0.36 \\
\hline 13 & 4 & 0.58 & 0 & 1.03 & 0 & 1.05 & 0 & 0.95 & 0 & 0.90 \\
\hline 14 & 4 & 0.35 & 0 & 0.95 & 0 & 0.99 & 0 & 0.86 & 0 & 0.79 \\
\hline 15 & 5 & 0.33 & 0 & 0.88 & 0 & 0.80 & 0 & 0.79 & 0 & 0.66 \\
\hline 16 & 6 & 0.22 & 0 & 0.93 & 0 & 0.99 & 0 & 0.85 & 4 & 0.45 \\
\hline 17 & 5 & 0.17 & 0 & 0.99 & 0 & 0.97 & 0 & 0.91 & 1 & 0.86 \\
\hline 18 & 4 & 0.32 & 0 & 1.02 & 0 & 1.00 & 0 & 0.89 & 0 & 0.92 \\
\hline 19 & 6 & 0 & 1 & 0.76 & 1 & 0.77 & 1 & 0.65 & 3 & 0.53 \\
\hline 20 & 3 & 0.45 & 0 & 1.10 & 0 & 1.01 & 0 & 0.93 & 0 & 0.93 \\
\hline 21 & 6 & 0.22 & 0 & 0.93 & 0 & 0.99 & 0 & 0.85 & 4 & 0.45 \\
\hline $\begin{array}{l}\text { Median/ } \\
\text { Mean }\end{array}$ & 5 & $\begin{array}{l}0.33 \pm \\
0.18\end{array}$ & 0 & $\begin{array}{l}0.90 \pm \\
0.16^{*}\end{array}$ & 0 & $\begin{array}{l}0.91 \\
\pm 0.1 \\
3^{*}\end{array}$ & 0 & $\begin{array}{l}0.85 \pm \\
0.16^{*}\end{array}$ & 0 & $\begin{array}{l}0.75 \\
\pm 0.2 \\
1 *\end{array}$ \\
\hline
\end{tabular}

2 RCC, Rutherford Clinical Classification. Least significant difference $t$-test, $* P<0.05$ (both

3 compared with $\mathrm{ABI} / \mathrm{RCC}$ before treatment). The median value is for $\mathrm{RCC}$, and the mean value is 4 for ABI, which were presented as mean \pm standard deviation. 